\title{
TIME ALLOCATION TO TOPICS IN A COURSE OF STUDY*
}

\author{
Maria NOWAKOWSKA** \\ Machine Intelligence Institute, Iona College, New Rochelle, NY 1080I, U.S.A. \\ James COX \\ Department of Psychology, University of North Carolina, Durham. NC. U.S.A. \\ Manfred KOCHEN
}

Mental Health Research Insritute. The University of Michigan, Ann Arbor, MI 48109, LiS..4.

Communicated by F.W. Roush

Received I May 1984

The paper presents a stochastic method of optimization of a curriculum based on a nell mode! of the education process, where sequential teaching may cause non-sequential learning.

Key words: Knowledge; stochastic; enlightenments; densities; probability.

\section{Introduction}

A person's knowledge grows as a result of his learning experiences. Some of these experiences are carefully structured. But there are many sources of such experiences. In formal education, teachers and books are the most common source. A problem common to many learning situations with diverse sources concerns the allocation of time. Relatively little is known about good ways to do that, despite its practical and theoretical importance.

Our primary object of inquiry is a randomly chosen student $S$ who exposes himself to various learning experiences in order to make discoveries, thus adding to his knowledge. We call the elementary discoveries he makes for himself 'enlightenments'. These may be known to everyone else, but it is an increment of knowledge to him.

To formulate a tractable problem, we consider a one-semester course with many sections, each taught by another instructor. We assume that the syllabus is given and organized into $n$ topics, $T_{1}, \ldots, T_{n}$. Each instuctor is expected to spend $t_{i}$ hours on topic $T_{i}$, so that the total number of hours is $K$. We ask what $t=\left(t_{1}, t_{2}, \ldots, t_{n}\right)$

* This study was initiated by Dr. Nowakowska as a consultant to a study on dynanics in the organization of scientific specialities, M. Kochen, Project Director, supported by NSF IST 800 7433. J. Cor worked on this project during July-August 1983.

** On leave from the-Institute of Philosophy and Sociology, Polish Academy of Sciences, Warsaw. 
should be so that $t_{i}>0, i=1, \ldots, n, \sum_{1-1}^{n} t_{i}=K$ and performance on a final examinanion is maximum.

The model to be analysed assumes that:

(1) the process of acquiring knowledge is stochastic;

(2) students differ in their abilities;

(3) teachers differ in their talents:

(4) performance of the final exam accurately reflects how much knowledge has been acquired.

The purpose of this analysis is to conceptualize this kind of learning process and to use a computer simulation to determine an optimal strategy in some situations.

\section{Assumpptions of the model}

We assume that topics $T_{1}, T_{2}, \ldots, T_{n}$ are taught in the order listed. The knowledge of the student at time $t$ is represented by the state vector

$$
x(t)=\left(x_{1}(t), \ldots, x_{n}(t)\right),
$$

where $x_{i}(l)$ is the number of enlightenments in topic $T_{i}$ that occurred prior to time $t$.

It will be assumed that $\boldsymbol{x}(t)$ is a Markov process, with elementary transitions consisting of an increase of one of the coordinates by 1 . The initial state is $x(i)=(0, \ldots, 0)$.

To describe the transition intensities, suppose that at time $t$ the state of the process is $x(1)$. and that topic $T_{A}$ is taught. Then we have

$$
P\left\{x_{i}(l+h)=x_{i}(t)+1 \mid x(t)\right\}= \begin{cases}\lambda_{k}(x) s i h+o(h) & \text { if } i=k . \\ \mu_{i k}(x) s=h+o(h) & \text { if } i<k . \\ 0 & \text { if } i>k .\end{cases}
$$

The last row in formula (2) implies th: enlightenments may concern only the topic actually taught, or topics that were taught previously, but not the topics in the future parts of the course.

Symbols $s$ and $z$ stand, respectively, for the student's ability and the teacher's talent.

Thus, furmula (2) asserts that the ability $s$ of the student and talent $=$ of the teacher are (a) representable as a single numiner, independent of the topic, and (b) enter in a multiplicative fashion.

As regards (a), it would be perhaps more appropriate to represent the ability of the student as a vector $\left(s_{1}, \ldots, s_{n}\right)$, with $s_{i}$ being his predisposition to the $i$ th topic (and similarly for the teacher's talent). However, one may hope that a representation as a single number (somewhat akin to Thurstone's mental cepacities, such as spatial aptitude, for example) does not constitute too severe a restriction.

On the other hand, part (b) is not really binding: as long as one is willing to accept that a more talented student, or a student taught by a more talented teacher, will 
have a higher rate of enlightenments, the multiplicativity may always be attained by appropriate scaling. That is to say, if (other conditions being equal) student $A$ has twice as high enlightenments rate as student B, one can assign to him ability $s_{\text {A }}$ equal $2 s_{B}$.

It remains to specify the functions $\lambda_{k}(x)$ and $\mu_{i k}(x)$. The first of them is defined as

$$
\lambda_{k}(x)=\frac{\left[\alpha_{k}+x_{k}(t)\right]\left[N_{k}-x_{k}(t)\right]}{\gamma D_{k}} \mathrm{e}^{h \mathrm{Q}}
$$

wilere

$$
Q=\sum_{i=1}^{k} c_{i k}\left(x_{i}(t)-v_{i} / 2\right)
$$

The interpretation of these formulas, and the meanings of symbols used, are as follows. Formula (3) asserts that the rate of increase of $x_{k}(i)$ is proportional to the product $\left[\alpha_{k}+x_{k}(t)\right]\left[N_{k}-x_{k}(t)\right]$. which implies that growth is roughly logistic, with $N_{k}$ being the maximal possible number of enlightenments in topic $T_{k}$. Here $\alpha_{k}$ is some constant that makes it possible for the process of enlightenments in topic $T$, to start.

Next, $y$ and $h$ are scaling constants, while $D_{k}$ is a measure of difficulty of topic $T_{k}$, so that the rate of enlightenments is lower for more difficult topics.

Finally, the factor $\mathrm{e}^{\text {hQ }}$ plays the following role. The sum $Q$ given by $(t)$ reflects the overall level of knowledge about topics $T_{1}, \ldots, T_{2}$, acquired previously, with differences $x_{i}(t)-N_{i} / 2$ being positive or negative, depending on whether more than half of possible enlightenments in topic $T_{i}$ occurred or not. Thus, $Q$ is a weighted sum of acquisition levels of previous topics, where the weight $c_{i}$ reflests how helpful a good knowledge of topic $T_{k}$ is in speeding up the ratc of enlightenments in topic $T_{k}$ (or alternatively, how much the lack of knowledge of topic $T$ impairs the rate of enlightenments in topic $T_{k}$ ). Consequently, Wcights $c_{2}$ reflest the internal structure of the domain taught.

Next,

$$
\mu_{i k}(x)=\alpha \beta^{k-i} \frac{\left[\alpha+x_{i}(t)\right]\left[N_{i}-x_{i}(t)\right]}{\gamma D_{i}} \exp \left\{h c_{i k}\left[x_{i}(t)-x_{i}-2\right]\right\} \text {. }
$$

To see the effects of this assumption, observe that the growth of $x_{i}(t)$. at the time when topic $T_{k}$ is taught, is again approximately logistic, impaired or enhanied by the level of difficulty $D_{1}$ of topic $T_{i}$, and acquisition level $x_{i}(t)-X_{i} \geq$ of topic taught. Here again, $\varrho$ is a scaling factor, while $\beta^{k-1}$ reflect 'memory loss', depen ding on the number of topics $T_{i}$ taught between $T_{:}$and $T_{i}$.

The choice of exponential factors in formulas (3) and (5) is admittedly arbitrary. If $h$ is small enough, the last factor in $(5)$ may be written as $1+h c_{i}\left(x_{i}(t)-1,2\right]$. which illustrates how the "excess' or 'defficiency" in knowledge of the $k$ th topic, as measured by $x_{k}(t)-N_{k} / 2$, enhances or inhibits the rate of enlightenments, yet preserves the crucial condition that the rate must be non-negative.

As regards the student's abilities and the teacher's talents, one has to assume that they vary according to some probability law. Many choices are possible here: 
in particular, one can assume that $s$ and $z$ have gamma distributions, with density of the form

$$
g(x)=\frac{a^{r}}{\Gamma(r)} x^{r-1} \mathrm{e}^{-a x}, \quad x>0,
$$

where $a$ and $r$ are the scale and shape parameters (possibly different for students and teachers). Consequently, the mean ability (talent) is $r / a$ and the variance is $r / a^{2}$.

This is not a very restrictive assumption, as long as one is willing to accept that each of the above talent distributions is unimodal: the family of gamma distributions is sufficiently rich to provide a reasonable approximation to many 'plausible' unimodal distributions. In particular, by choosing $a$ and $r$ one can set the mean and the coefficient of variation $1 / r^{1 / 2}$ at any desired values.

\section{Final exam}

The total knowledge acquired by the student at the end of the course is the criterion to be maximized by the choice oi the decision variable $t=\left(t_{1}, \ldots, t_{n}\right)$. This tola! knowledge is represented by $x(K)=\left(x_{1}(K), \ldots, x_{n}(K)\right)$, where $K=\sum_{i=1}^{n} t_{i}$ is the rotal number of hours spent on all topics.

To formulate a criterion to be optimized by the choice of $t$, we may proceed as follows.

The knowledge $x(K)$ acquired by a student is a random entity, with distribution depending on the decision variable $t$; it also depends on the student's ability $s$, and on the talent $z$ of the teacher. Thus, we may write

$$
P\left\{x_{1}(K)=k_{1}, \ldots, x_{n}(K)=k_{n}\right\}=P\left(k_{1}, \ldots, k_{n} \mid s, z, t\right) .
$$

Typically, the vector $\left(k_{1}, \ldots, k_{n}\right)$ is not observable. Instead, one may observe the score $\xi$ on the final exam, which may be regarded as a random variable, whose distribution is monotonic with respect to $x=\left(k_{1}, \ldots, k_{n}\right)$, in the sense that

$$
E\left(\xi \mid x_{1}(K)=k_{1}, \ldots, x_{n}(K)=k_{n}\right)
$$

is an increasing function of each of the arguments $k_{1}, \ldots, k_{n}$.

We have therefore the expected final score for a student with ability $s$ taught by a teacher with talent $z$ :

$$
E(\xi \mid s, z, t)=\underset{\left(k_{1}, \ldots, k_{n}\right)}{\sum} E\left(\xi \mid x_{1}(K)=k_{1}, \ldots, x_{n}(K)=k_{n}\right) P\left(k_{1}, \ldots, k_{n} \mid s, z, t\right) .
$$

The problem may now be formulated as follows:

Find $t_{1}, t_{2}, \ldots, t_{n}$, subject to constraints 


$$
t_{i}>0, \quad i=1, \ldots, n, \quad \sum_{i-1}^{n} t_{i}=K
$$

such that

$$
E(\xi \mid t)=\int_{0}^{\infty} \int_{0}^{\infty} E(\xi \mid s, z, t) g_{1}(s) g_{2}(z) \mathrm{d} s \mathrm{~d} z
$$

is maximum (here $g_{1}$ and $g_{2}$ are the densities of $s$ and $z$, given by (6)).

\section{Analysis}

Two entities that are crucial here are the distribution (7) of the final attained knowledge, and the expected score (8) on the exam, given the final knowledge. The latter quantity, incidentally, may depend on the student's ability $s$, if the exam requires not only knowledge, but also some creativity in applying it.

As regards (8), it is relatively easy to postulate a reasonable form of the relation between the expected final score on the exam, and the knowledge of particular topics. The exam typically has a number of problems, each concerning one or more topies, and the probability of a correct answer may be postulated to be some simple function (e.g. logistic) of the number of enlightenments in the relevant topics.

As regards (7), the explicit solutions do not appear easily obtainable; however, one can easily simulate the process of knowledge acquisition directly from the hypotheses of the model, thus obtaining empirical access to distribution (7), or to criterion (11). The simulation algorithm is based on the following theorems.

Theorem 1. Let $t$ be such that

$$
t_{1}+\cdots+t_{k-1} \leq t<t_{1}+\cdots+t_{k},
$$

so that topic $T_{k}$ is taught at $t$. Suppose that the state of knowledge of the student at $t$ is $\boldsymbol{x}(t)=\left(x_{1}(t), \ldots, x_{k}(t), 0, \ldots, 0\right)$. Then the probability that next enlightenments occurs before time $\tau$ such that $t \leq \tau<t_{1}+\cdots+t_{k}$ equals

$$
1-\exp [-(\tau-t) \operatorname{sz} V(x)],
$$

and with probability

$$
\exp \left[-\left(t_{1}+\cdots+t_{k}-t\right) \operatorname{sz} V(x)\right]
$$

there will be no enlightenments occurring while topic $T_{k}$ is iaught, where

$$
V(x)=\lambda_{k}(x)+\sum_{i=1}^{k-1} \mu_{i k}(x)
$$

Theorem 2. If an enlightenment occurs while topic $T_{k}$ is taught, it concerns topic $T_{k}$ with probability 


$$
p_{k}=\lambda_{k}(x) / V(x)
$$

and it concrens a previously taught topic $T$, with probability

$$
p_{i}=\mu_{i k}(x) / V(x)
$$

Proof. Let $P(\tau)=P(\tau ; t, x)$ be the probability of no change of state $x$ between $t$ and $\tau$. Then for $h$ such that $\tau<t+h<t_{1}+\cdots+t_{k}$ we have

$$
\begin{aligned}
P(\tau+h) & =P(\tau)\left[1-s z \lambda_{k}(x) h-s z \sum_{i=1}^{k-1} \mu_{i k}(x) h\right]+o(h) \\
& =P(\tau)[1-s z V(x) h]+o(h),
\end{aligned}
$$

by assumption (2). Dividing both sides by $h$, and letting $h \downarrow 0$, we obtain

$$
P^{\prime}(\tau)=-s z V(x) P(\tau),
$$

which yields assertions (13) and (14). To prove Theorem 2, observe that if a $1 \mathrm{r}$. 1sition occurs, then the probabilities of various types of transitions are given : the rolative contributions to $V(x)$ from intensities $\lambda_{k}(x)$ and $\mu_{i k}(x)$.

\section{Discussion}

Despite the importance of the problem, there is so far no research on optimality of the time allocation for curriculum structuring. However, certain aspects of such research have been investigated, and one can find in the literature several relevant papers. For example, there were attempts at mathematical modeling of student testing for instructional purposes; for example, in Pinsky (1970), psychometric methods were applied for instructional improvement, and for selection of test items (including a selection algorithm), used for estimation of the desired parameters.

The present discussion will concern, first of all, the assumptions underlying the mode! and their plausibility, and secondly, the problems of estimation of the parameters needed as input data in the simulation program aimed at optimizing the allocation of teaching times.

The main assumption that the accumulation of enlightenments can be counted requires some explanation, as does the assumption that learning takes place primarily in this way.

In practice, many high-level geometry studints make no discoveries of their own at all, but merely memorize enough of what think will be on the exam to pass i1. Our use of 'learning' in this paper is restricted, so as to exclude this. A student who learns in our sense might discover, or come to see why, the theorem of Pythagoras is useful, important or true, or how to construct a line whose length is the square root of a given line. When we count the enlightenments by giving the same weights to (re)discovering the Pythagorean theorem and to the fact that 3, 4 and 5 are lengths of the sides of a right-angle triangle is a distortion, for the former is of much greater importance than the latter. 
In fact, however, one may interpret the notion of an enligittenment in two ways. One is to regard an enlightenment as an assimilation of a piece of knowledge (e.g. memorizing the theorem of Pythagoras), without grasping the possible relationships with other pieces of knowledge. In this case, $\boldsymbol{x}_{i}(t)$ could perhaps be operationalized, through listing all pieces of information deemed relevant, and checking how many of them are known to the student at the time of taking the exam.

It is possible, however, to view the concept of enlightenment differently. The starting point here is the fact that students do not accumulate pieces of knowledge one at a time, but assimilate them into knowledge structures that make our minds prepared to accept them; at the same time, these assimilations enrich these knowledge structures and change their preparedness for new potential pieces of knowledge. Accordingly', we may view enlightenments as instances of grasping some previously not realized relationships between two distinct pieces of knowledge. Using a simple example, the enlightenments would be not mere learning of the theorem of Pythagoras, and of the fact that the triangle with sides 3,4 and 5 is a right-angled one, but the realization that these two facts are closely related.

In this interpretation, the number $x_{i}(t)$ of enlightenments is not easily observable; it may, however, be postulated that it is monotonically related to the expected score on the exam.

In our model, the intention was to frame the assumptions so as to cover the second interpretation.

This is closely related to our assumption that the process of cacumulation of enlightenments is a Markov process, with only' 'upward' transitions possible.

As regards the second of these assumptions, it means that once an enlightenmens occurs, it is never forgotten. This is, of course, an idealization of reality: the students often forget the material they learned. However, while it may be easy to forget an isolated piece of knowledge, forgetting something that resulted from a sudden instance of understanding is much less likely. Consequently, if one accepts the second of the above interpretations of enlightenments, then the assumption tha: they are irreversible may not be very much far-fetchcd.

The Markovian character of the process of enlightenments implies ihat the probability of the next enlightenment is independent of the time that elapsed from the la:.: enlightenment. This neglects the effects of loss of zeal in studying due to discouragt:ment, etc. However, the fact that Markov process is 'memoryless' (in the sense that given the present, the past values have no influence on probabilities of events in the future) does not imply that the model assumes lack of memory of student. In firct, the specific assumptions about the enlightenment rates describe in some sense the student's memory and its role in learning, including some features of forgetling (through making enlightenments in temporally distant topics gradually less imp $\mathrm{O}^{-}-$ tant for new enlightenments).

Now, the specific assumptions concern the functional form of the enlightenment rates, given the topic that is actually taught and the student's knowledge of current and previous topics. 
Basically, these rates imply a logistic growth in each topic, modified by the state of knowledge of other topics. These assumptions do not appear directly testable. However, the general logistic character may be defended on intuitive ground: at the beginning, the knowledge 'builds on itself' in a more or less exponential fashion, as one grasps various easily accessible relationships. At later stages the growth rate do:lines, as one is required to grasp less immediate relationships.

There is probably little doubt that the state of knowledge of other topics may have an enhancing or inhibiting effect on enlightenment rates. Whether or not the modifying factor is indeed of the exponential form postulated in the model is an open question, and it is not clear whether the assumption is empirically testable. However, the functional form of enlightenment rates appears sufficiently flexible to approximate intuitively acceptable alternative functions.

A question that requires careful consideration here is as follows. At present, it is assumed that the state of knowledge of a given topic intervenes through the term $x_{i}(I)-N_{i} / 2$. Thus, if less than one-half of all possible enlightenments occurred in a topic, we bave $x_{i}(t)<N_{i} / 2$, and the term is negative, having therefore an inhibiting effect on the rates of enlightenments. If more than half possible enlightenments occurred, $x_{i}(t)-N_{i} / 2$ is positive, and has therefore an enhancing effect. While it is reasonable to postulate an effect of such a form, there is little justification for selecting $\boldsymbol{N}_{i} / 2$ as the critical threshold. It is quite possible that these thresholds vary from topic to topic, and their choice should be made after analysing the subject taught.

\section{Fstimation}

:o use the simulation program, one needs to have estimates of certain parame! crs. First, the maximal possible numbers of enlightenments in various topics can be Jetermined from the subject-matter analysis: as an approximation of $N_{i}$, one may count the number of items on a list of all possible ideas that one can expect the top students to know and understand.

An experienced instructor could also provide as iessments of the relative difficulty levels $D_{i}$ of topics. These (as well as the coefficients $c_{i k}$ ) may be scaled arbitrarily, since it is only their ratios that matter (the masnitudes become absorbed by scaling factors).

As regards coefficients $c_{i k}$, they again may be assessed by experienced instructor $\dot{\text {. }}$. Roughly, $c_{i k}$ may be measured by the number of items on a list of instances when some facts about the $i$ th topic are recalled or used in reaching the $k$ th topic.

As regards difficulties of topics, one could also try an empirical approach in the following way. Suppose we devise tests covering various topics in the course. Assume that a test for the $k$ th topic contains $\boldsymbol{R}_{\boldsymbol{k}}$ (binary, say) items, and the score of a student is the number of items that he answers correctly (one could, of course, modify this approach by considering partial scores, etc.). The data have the form of a matrix $\left[i_{i k}\right]$, where $z_{i k}$ is the score of the $i$ th student on the $k$ th test (i.e. concernirig the $k$ th topic). This matrix allows us then to gain some insight into the rela- 
tive difficulties of topics by analysing the average scores in various topics (averaging over the students). However, such data may serve at best only as a guideline, since the scores depend not only on the difficulties (which we wani to measure), but also on the attained knowledge. Similarly, the averages of scores $z_{i k}$ across the topics may provide some information about the distribution of students' abilities. Here again, however, the data are confounded by the attainment levels, which cannot be separated from abilities.

It remains to outline the method of assessing the values of the remaining parameters, namely the scaling values $\gamma, h, \varrho$ and $\beta$, as well as the vector of $\alpha_{k}$ 's, and the parameters of the distribution of the teacher's talents and the student's abilities.

Here the idea is to use the knowledge of learning rates, either known from instructors' experience or acquired from carefully designed experiments.

Suppose that the difficulties $D_{k}$ and coefficients $c_{i k}$ have already been chosen, and the values $N_{k}$ are known. The scaling of $D_{k}$ and $c_{i k}$ is, as mentioned, arbitrary (e.g. one may assign $D_{k}=100$ to the most difficult topic, and smaller values to the remaining topics, so as to express the ratios of difficulty).

Suppose now that we take an average student's ability and an average teacher's talent to be 100 (say). To determine the values of $\alpha$ and $\gamma$ imagine that an average student taught by an average teacher acquired about one-half of the possible knowledge of previous topics, and that at time $t$ he starts to learn a new topic with difficulty $D_{k}$, We have therefore $x_{j}(t)=N_{j} / 2$ for $j<k$, hence $Q=0$ and $x_{k}(t)=0$. The enlightenment intensity for the $k$ th topic is therefore, using (3):

$$
\lambda_{k}=\frac{\alpha N_{k}}{\gamma D_{k}} 1,00 \times 100 ;
$$

hence, the average time until the first enlightenment in the $k$ th topic is $1 / \lambda_{\alpha}$. If we now find (e.g. from some experiment or from the instructor's experience) that an average student, taught by an average teacher, with average knowledge of previous topics, should have his first instance of understanding the topic of difficulty $D_{k}$ after, say, 20 minutes of learning, then $1 / \lambda_{k}=0.33$ (hours), which gives an assessment of the ratio $\alpha / \gamma=0.3310^{4} N_{k j} / D_{k}$. Suppose now that the same student, at some later time, acquired about one-half of the knowledge of the $k$ th topic, while it is still being taught. His learning rate is now fastest, and we can expect, say, the average time between enlightenments to be only 10 minutes (i.e. about six "graspings" of the material per hour of learning). We have now

$$
\lambda_{k}=\frac{\left(\alpha+N_{k} / 2\right) N_{k} / 2}{\gamma D_{k}} 10^{4},
$$

and, taking $1 / \lambda_{k}=1 / 6$, we obtain a second equation for $\alpha$ and $\gamma$.

After choosing $\alpha$ and $\gamma$ from the knowledge of average times to enlightenments for an average student with average previous knowledge, we can assess $h$ by considering how much improvement is gained by previous knowledge. Thus, suppose that we have two students, with the same abilities, taught by the same teacher, one with the average knowledge of past topics (so that $x_{i}(t)$ is about $N_{i} / 2$ for all past topics), and the other with a 'good' knowledge, say $x_{i}(t)=0.75 N_{i}$. The value $Q$ for 
The first student is $\mathbf{0}$, and for the second it may be computed knowing the constants $c_{i k}$. If the rate of enlighienments of the first student is $\lambda$, that of the second is $\lambda e^{h Q}$, so that ratio of the rates, and hence the ratio of average inter-enlightenment times, is $\mathrm{e}^{\text {inQ }}$.

Similar reasonings, only concerning average enlightenment times in past topics, may lead to assessment of the constants $\varrho$ and $\beta$.

Finally, an assessment of the parameters of the distribution of teachers' talents and students' abilities may be obtained by considering the amounts of variability of leat ning results (known from experience; as the means are set at the value 100 , only one parameter needs to be determined for each of these distributions).

\section{Simulation}

Using Theorems 1 and 2, we may devise a computer simulation program that might allow us to find empirically the optimal allocation of teaching times to topics.

The flowchart (Fig. 1, pp. 287-288) concerns only the process of enlightenments (not the final exam), for given values of the student 's ability and the teacher's talent. The symbol $\zeta$ stands for a random number sampled from the uniform distribution on $[0,1]$.

The question arises as to what extent one can expect to obtain practically meaningful results by simulation, in view of the fact that the input parameters are assessed with some dose of subjectivity.

First, the estimates obtained from 'educated guesses' by experienced instructors yield a plausible range of parameter values. In other words, one may believe that hypothetical experiments would provide estimates of parameters that lie within the obtained range.

A simple sensitivity analysis will provide data about how much the results depend on errors in assessment of particular values of parameters.

Secondly, even if some of the parameters remain empirically inaccessible, the simulation performed for various plausible combinations of parameters could provide information relevant for time allocation. For instance, common sense suggests that more difficult topics should be allocated more time (other conditions being equal). It is not clear, however, whether a topic that is twice as difficult should be allocated twice as much time, or the relationship here is not of the direct proportionality. Here one could collect simulated teaching effects under various time allocations, and for various plausible combinavions of parameters. Any feature that remains invariant under variation of parameters may then be taken as a law, applicable in practice, even in situations when the parameters are unknown.

Of particular interest here will be to analyse optimal allocation of teaching times in its relation to:

varying difficulties of topics;

varying amounts of material in topics (i.e. different maximal numbers of enlightenments $\boldsymbol{N}_{k}$ );

variability in students' abilities (e.g. screening or selection of students will tend to decrease such variability). 


\section{Comments}

The paper presents not only an economical way to structure a curriculum for educational purposes, but primarily a way to study a very complex educational process, allowing for controlled changes of content and also for tims allocated to topics. This method may be valuable both in unification and individualization of education, in the case of normal, mentally retarded, or especially talented pupils.

One could also consider introducing a modern type of curriculum which combines, in various degrees, traditional and computer instruction. In the near future. because of the development of educational sottware, the latter may contain progressively more elements of self-teaching combined with special systems of cognitive and social rewards by the instructor or the group. Such flexible education systems with known properties, where the teacher's role would consist more in chosising and supplying the educational means (as produced by educational centers), d:veloping the motivational aspects of students, and explaning more difficult material, would allow an increase in the educational and social role of especially talented instructors at all levels, and also it would allow the cost of education to be lowered. A shift in responsibility toward student-centered edacation would have a stimulating effect on developing initiatives in extramural education, supporting the curriculum.

It appears that in the future the comprehensive studies of the educational processes will play an increasingly important role; in particular, this will soncern the optimizing properties, as regards the temporal allocation of tasks (topics). logical and formal structures of curriculi, and the interdependance of topics.

The approach suggested abondons the traditional sequential treatment in learning: it introduces a new interpretation and model of the phenomenon of learning which takes into account not only the discrete increments of knowledge and understanding that appear during learning, but also increments of knowledge and understanding of the material that already has been covered and mastered to some degree. This complicated process of an increase in knowledge is modeled by taking into aciount the logical and content-dependent relations between units of the surriculum, and their varying levels of difficulty. The increments in understanding the material actually taught, as well as that which has been taught before, are treated in the model as a certain multidimsnsional stochastic process, whose parameters differ for various students and also for various teachers (or software). This new way of treating learning as a process with returning, reinterpretation and improvements of understanding of old topics during the study of subsequent ones, may by itsc if constitute a starting point for the construction of new types of measurement of the quality of learning, and also for the sonstruction of curricula and the study of their properties. The model suggests the importance of returns and new interpretation of previous material in educational process, and abandoning the purely sequential ways of thinking about structuring curricula.

Perhaps, especially for less talented children, the returns to previous units in the curriculum may elicit awareness and also increase the level of understanding. It appears that the less able and unintelligent the student, the mor: often it is necsessary to return and restructure the previous units of the curriculum. This is especially im- 
portant in creating software for less talented children. It is not necessary to emphasize the significance of optimization of the temporal aspects, and the economical aspects have already been mentioned. But still another point is the steadily increasing amount of more and more difficult knowledge, which new generations of children must absorb in about the same amount of time, if educational process is not to interfere with normal harmonious biological and psychological development.

As emphasized above, the responsibility for study should, whenever possible, be shifted towards the student. The aim of an educational system should, to a large extent, be to supply interesting, cognitively valuable, and ethically sensitive curricula, with reasonably embedded self-teaching programs. At the same time it should preserve the initiating, motivating and controlling role of the teacher. The latter should elicit critical and anti-stereotypic thinking.

For especially talented children, one should encourage the self-rewarding development of interests and cognitive search, rather than through competition and threat of ioss of future economic position, job, etc.

The approach suggested does not lead to overly rigid and stereotype curricula: the point is to supply curricula with some known properties, but which are also flexible (with possibilities of transformations, additions, and open slots for innovations and discussions). This will probably require some special training for teachers on how to stimulate the interaction between student and computer.

To sum up, special attention must be devoted to designing software in education. It may be necessary to create a federal institution which would produce such software, designed by qualified psychologists and educationists. It is equally important that the use of self-teaching methods and other technical innovations be supervised and have a group character, since it is the school that has to teach also intellectual cooperation and social behaviour - ethical and noble patterns of behaviour, namely reliability, righteousness, reflexive attitude towards himself and others in work and in emotional relations. Learning of ethical behaviour and philosophical reflexions on any of educational levels should be particularly encouraged, in view of anti-stress role of such knowledge.

\section{References}

P.D. Pinsky, A mathematical model of testing for insuructional management purposes, Conference paper, Annual Meeting of American Educational Research Association, Minneapolis (March 1970).

M. Powell, Time allocations: Teachers help determine urriculum, Educational Horizons $\$ 7,4(1979)$ 175-177.

Utah State University, Interactive videodisc for special educational technology: General report, Logan, UT (1982). 
SIMULATION FLOWCHART

$$
\begin{aligned}
& n_{1}, \ldots, N_{n} \\
& N_{1}, \ldots, 0_{n} \\
& 0_{1}, \ldots, t_{n} \\
& t_{1}, \ldots \\
& ! c_{i k} ! \\
& h, a, 2, \ldots, 0 \\
& z
\end{aligned}
$$$$
\text { INITIAL VALUES }
$$$$
\text { - NUMBER OF TOPICS }
$$$$
\text { OF ENLIGHTMENTS }
$$$$
\text { - Difficulty leVElS }
$$$$
\text { - allocated numbers }
$$$$
\text { OF HOURS }
$$$$
\text { - Structure matrix }
$$$$
\text { - PARAMETERS }
$$$$
\text { - STUDENT'S ABILITY }
$$$$
\text { - TEACHER'S TALENT }
$$

T IS THE TIME OF FINISHING CURRENT TOPIC
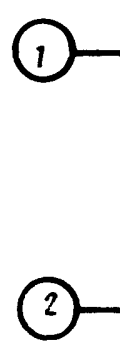

SETS INITIAL NUMEERS IF

ENLIGHTUENTS

$S$ IS THE RLASIAG TIIUE CF EAITICTYEST EEGIN LOCF

NEXT TOPIC

CALCULATES

INTENSITY.

Fig. 1 


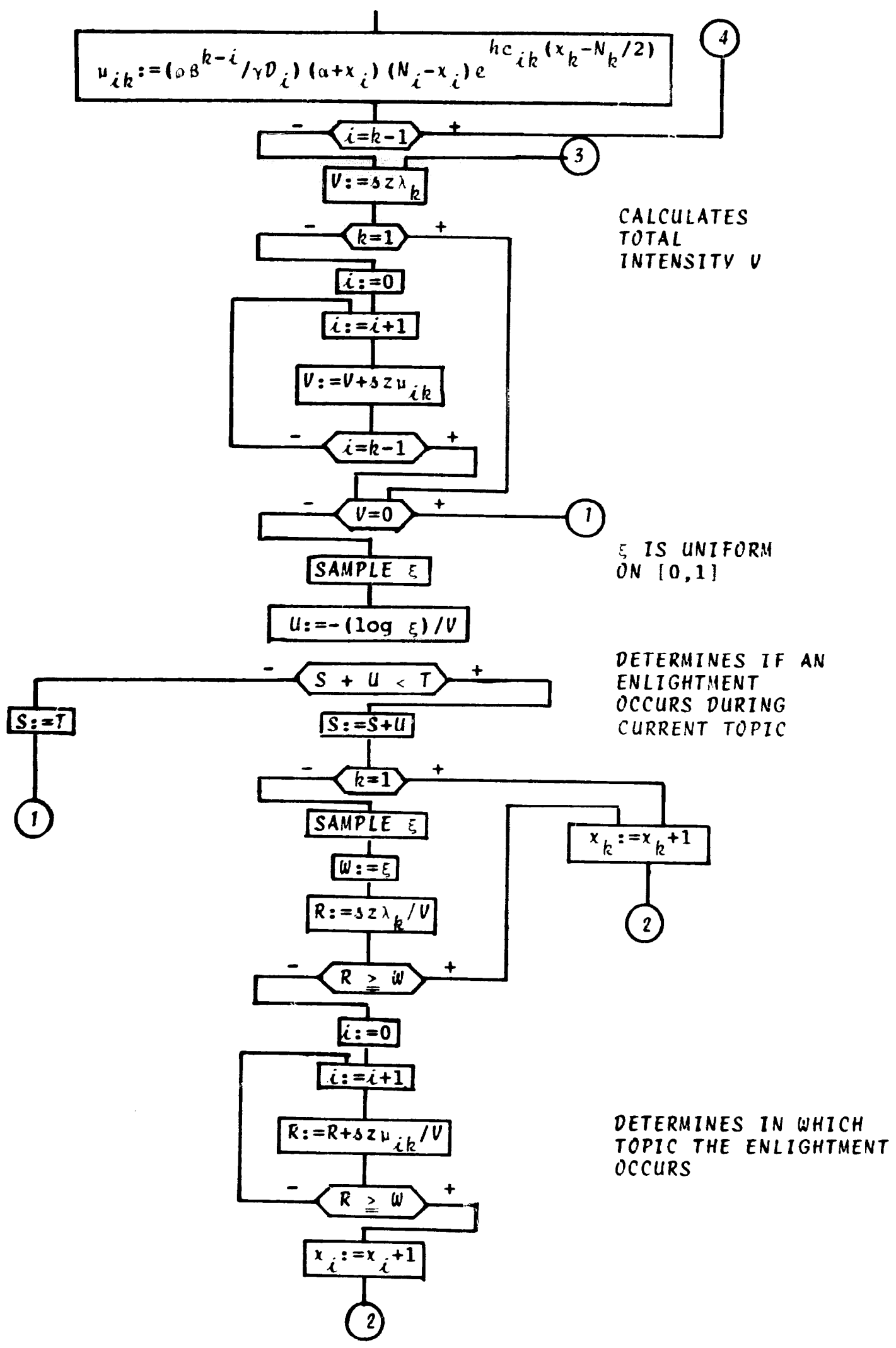

